Supporting informations for the manuscript:

\title{
An atomistic simulation of phase transitions and charge mobility for the organic semiconductor Ph-BTBT-C10
}

\author{
Alberto Baggioli, ${ }^{\dagger}$ Mosè Casalegno,${ }^{\dagger}$ Guido Raos, ${ }^{*}, \dagger$ Luca Muccioli, ${ }^{\ddagger}$ Silvia Orlandi, ${ }^{*},{ }^{\dagger}$ Claudio Zannoni*,$\hbar$ \\ $\dagger$ Dipartimento di Chimica, Materiali e Ingegneria Chimica "Giulio Natta", Politecnico di Milano, via L. Mancinelli 7, 20131 Milano, Italy \\ * Dipartimento di Chimica Industriale "Toso Montanari", Università di Bologna, viale Risorgimento 4, 40136 Bologna, Italy
}

Content:

Figure S1. Torsional potential for the Phenyl-BTBT dihedral. S2

Figure S2. MSD along the director for crystalline and smectic phases. S2

Table S1. Simulated values of $d, \tau_{1}, \tau_{2}, \tau_{3},\left\langle P_{2}\right\rangle, \psi_{4}, \psi_{6}$. S3

Figure S3. Molecular reorientation autocorrelation function. S3

Figure S4. GGA/DZ-based vs. B3LYP/DZ-based orbital couplings. S4

Figure S5. BLYP/DZ-based vs. B3LYP/TZ-based orbital couplings. S5

Figure S6. Piecewise exponential fit of $\sigma^{2}\left(r_{A A}\right)$. S6

Figure S7. Corrected orbital coupling probability distributions. S6

Figure S8. Hopping rate probability distributions. S7

Table S2. Site energy standard deviations. S7

Table S3. Hole Diffusivity as computed from KMC simulations $\left(\Delta \varepsilon_{\mathrm{pq}}=0\right)$. S8

Table S4. Standard deviation of hole Diffusivity $\left(\Delta \varepsilon_{\mathrm{pq}}=0\right)$. S9

Table S5. Hole Diffusivity as computed from KMC simulations $\left(\Delta \varepsilon_{\mathrm{pq}} \neq 0\right)$. S10

Table S6. Standard deviation of hole Diffusivity $\left(\Delta \varepsilon_{\mathrm{pq}} \neq 0\right)$. S11

Figure S9. Relative site occupancy probability distributions. $\mathrm{S} 12$

Figure S10. Average residence time probability distributions. S13

Figure S11. Hole mobility from uncorrected orbital couplings. S14 


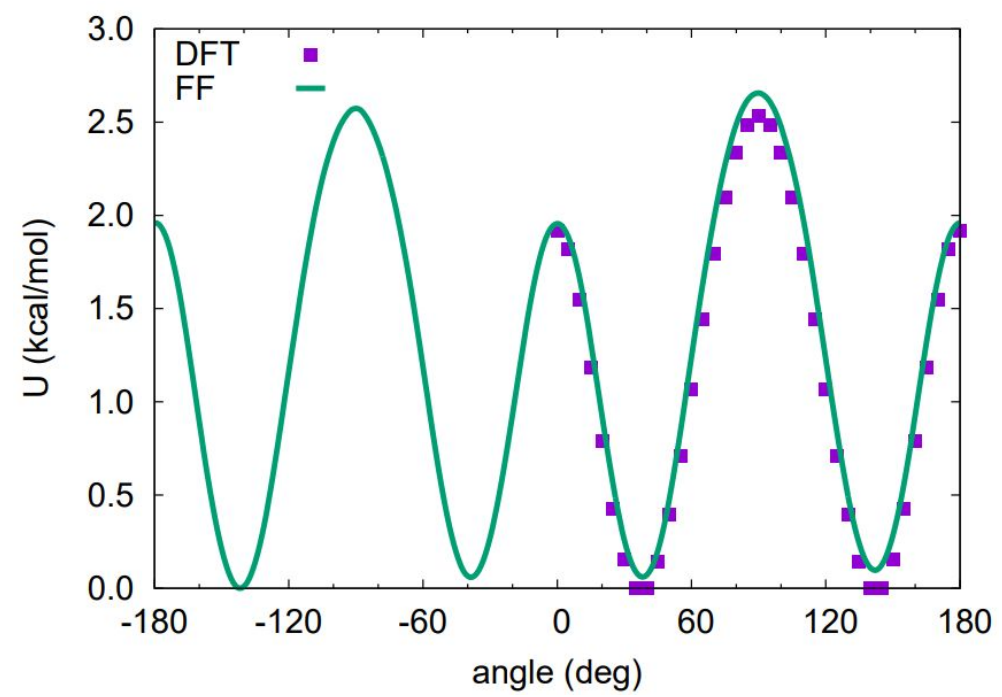

Figure S1. Torsional potential for the Phenyl-BTBT dihedral as obtained from DFT PBE0/cc-pVTZ calculations and from the molecular mechanics force field used in this work.

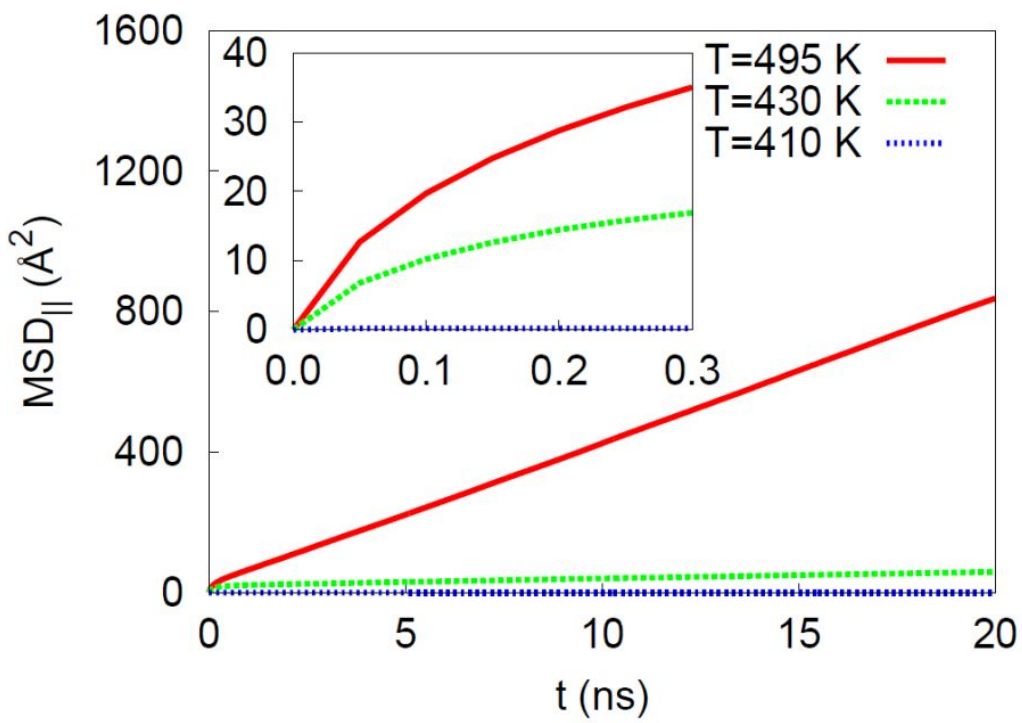

Figure S2. Mean square displacements along the director (i.e. across layers) for crystalline and smectic phases at selected temperatures in the interval 0-20 ns; in the inset, a zoom into the first $0.3 \mathrm{~ns}$. 
Table S1. Simulated values of layer spacing $d$, positional order parameters $\tau_{1}, \tau_{2}, \tau_{3}$, second rank orientational order parameter $\left\langle P_{2}\right\rangle$, and tetradic and hexatic order parameters $\psi_{4}$ and $\psi_{6}$ with respect to temperature.

\begin{tabular}{lccccccc}
\hline $\mathbf{T}(\mathbf{K})$ & $\boldsymbol{d}(\boldsymbol{\AA})$ & $\boldsymbol{\tau}_{\mathbf{1}}$ & $\boldsymbol{\tau}_{\mathbf{2}}$ & $\boldsymbol{\tau}_{\mathbf{3}}$ & $\left\langle\boldsymbol{P}_{\mathbf{2}}\right\rangle$ & $\boldsymbol{\psi}_{\mathbf{4}}$ & $\boldsymbol{\psi}_{\mathbf{6}}$ \\
\hline $\mathbf{4 1 0}$ & 26.0 & 0.95 & 0.69 & 0.43 & 0.99 & 0.82 & 0.62 \\
$\mathbf{4 1 5}$ & 26.0 & 0.94 & 0.68 & 0.42 & 0.99 & 0.80 & 0.61 \\
$\mathbf{4 1 8}$ & 26.1 & 0.93 & 0.68 & 0.43 & 0.99 & 0.80 & 0.64 \\
$\mathbf{4 2 5}$ & 27.6 & 0.78 & 0.36 & 0.11 & 0.90 & 0.05 & 0.02 \\
$\mathbf{4 3 0}$ & 27.6 & 0.76 & 0.26 & 0.11 & 0.90 & 0.05 & 0.02 \\
$\mathbf{4 4 0}$ & 27.7 & 0.69 & 0.26 & 0.08 & 0.89 & 0.05 & 0.03 \\
$\mathbf{4 5 0}$ & 27.7 & 0.70 & 0.23 & 0.06 & 0.88 & 0.05 & 0.03 \\
$\mathbf{4 6 0}$ & 27.6 & 0.69 & 0.23 & 0.06 & 0.88 & 0.05 & 0.03 \\
$\mathbf{4 7 0}$ & 27.6 & 0.66 & 0.21 & 0.06 & 0.86 & 0.05 & 0.03 \\
$\mathbf{4 8 0}$ & 27.7 & 0.60 & 0.21 & 0.06 & 0.84 & 0.05 & 0.03 \\
$\mathbf{4 9 0}$ & 27.8 & 0.57 & 0.17 & 0.06 & 0.82 & 0.06 & 0.03 \\
$\mathbf{4 9 2}$ & 27.8 & 0.57 & 0.16 & 0.05 & 0.81 & 0.06 & 0.03 \\
$\mathbf{4 9 4}$ & 27.8 & 0.58 & 0.16 & 0.05 & 0.81 & 0.06 & 0.03 \\
$\mathbf{4 9 5}$ & 27.8 & 0.52 & 0.15 & 0.05 & 0.78 & 0.06 & 0.03 \\
$\mathbf{4 9 6}$ & - & - & - & - & 0.0 & 0.06 & 0.03 \\
$\mathbf{4 9 7}$ & - & - & - & - & 0.0 & 0.06 & 0.03 \\
\hline
\end{tabular}

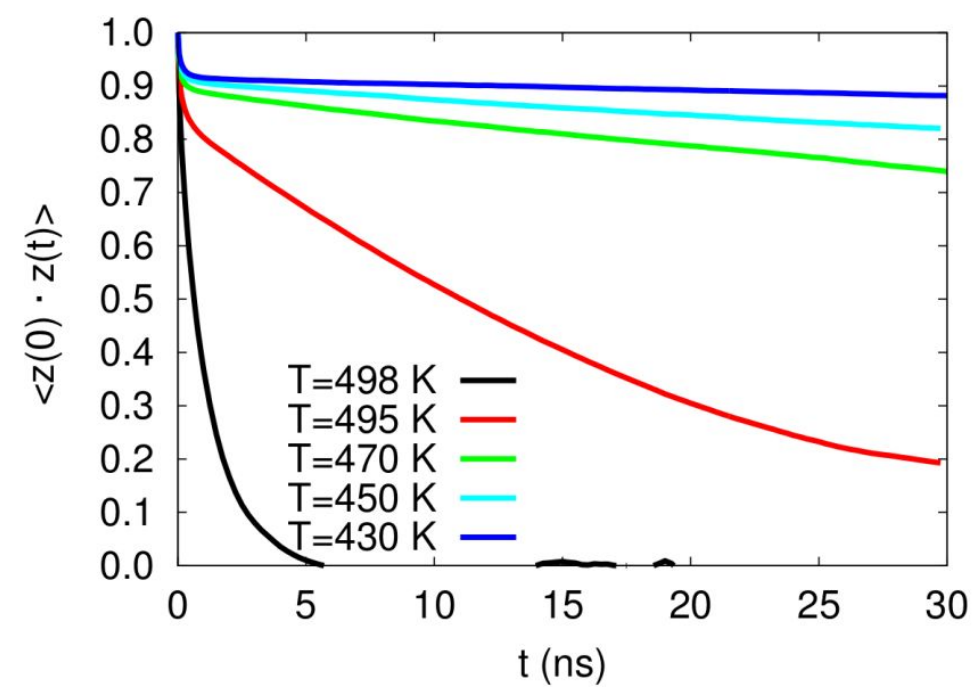

Figure S3. Autocorrelation function for reorientation around the molecular $\mathrm{z}$ axis at different temperatures within the regions of existence of smectic and isotropic phases. 

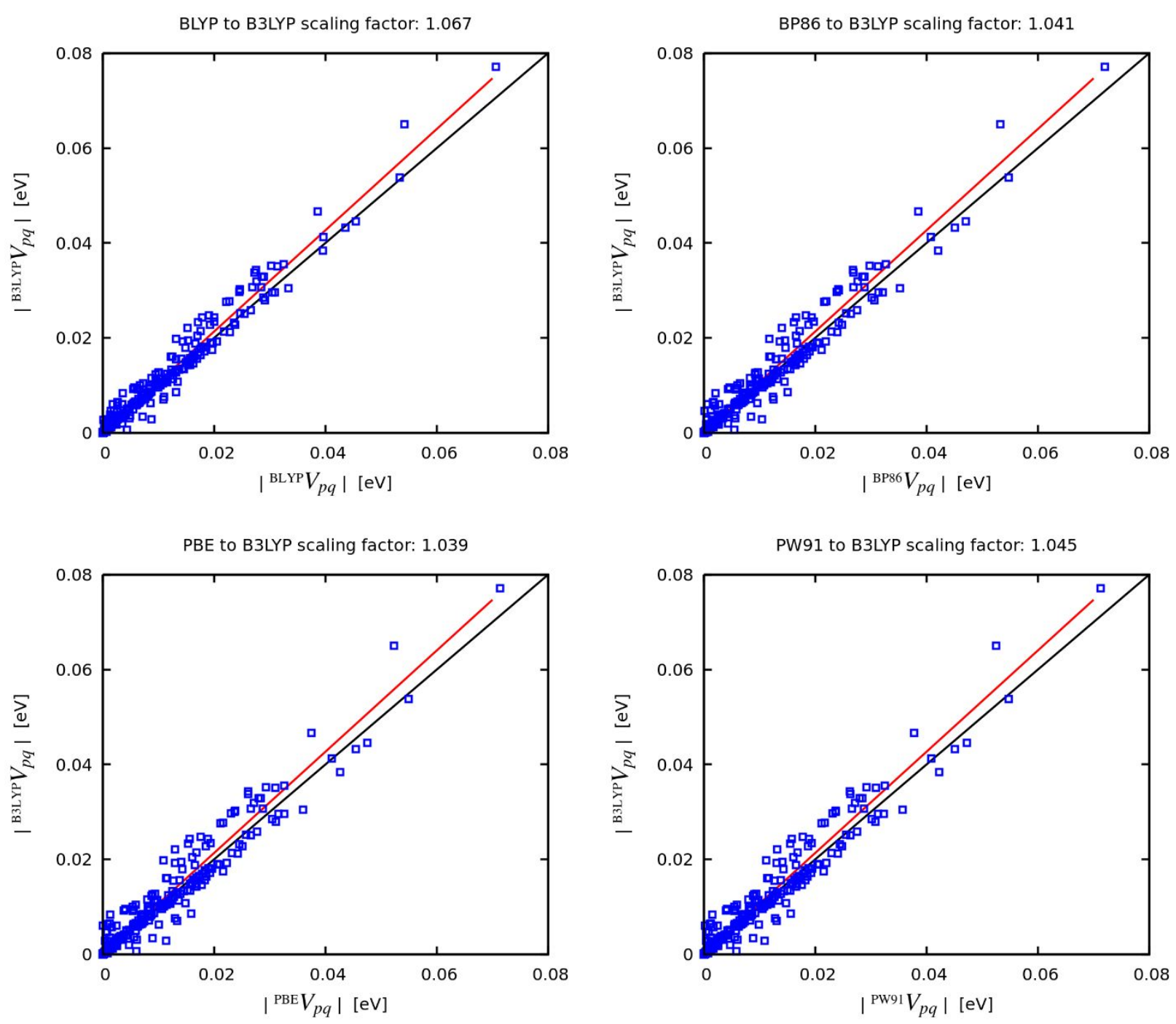

Figure S4. Scatter plots of GGA/DZ-based orbital couplings against a B3LYP/DZ reference. Fitting lines (red) are reported in each panel along with the corresponding scaling factors.

Two sets of 200 randomly picked Ph-BTBT-C10 dimers were extracted from MD snapshots obtained at $293 \mathrm{~K}$ and $490 \mathrm{~K}$, respectively, for a total of 400 points.

Orbital couplings were computed from hybrid generalized gradient-corrected (hybrid-GGA) B3LYP, and from pure gradient-corrected (pure-GGA) BLYP, BP86, PBE, and PW91 data. A double- $\zeta$ (DZ) basis set, Pople's 6-31G(d), was used throughout.

The set of $400\left({ }^{\mathrm{B} 3 \mathrm{LYP}} V_{p q},{ }^{\mathrm{GGA}} V_{p q}\right)$ points was then fitted over a line in the form $f(x)=a x$ in order to obtain linear scaling factors $a$ (reported on top of each plot). 


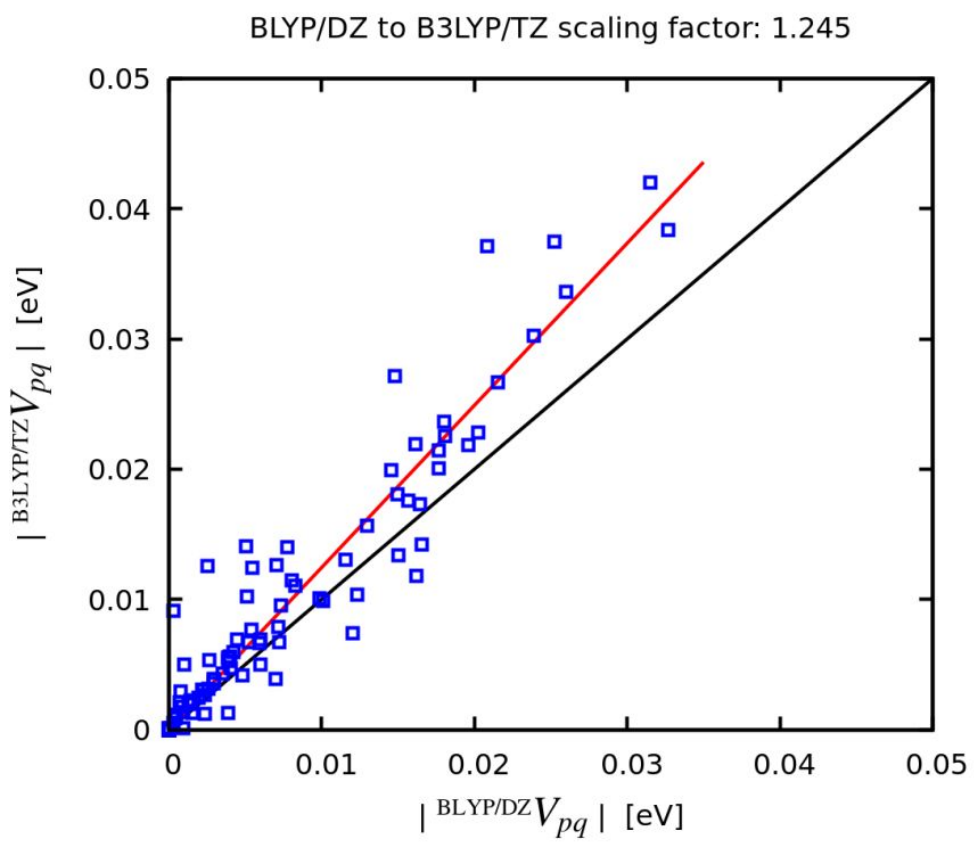

Figure S5. Scatter plot of BLYP/DZ-based orbital couplings against a B3LYP/TZ reference. Fitting line (red) and the corresponding scaling factor are reported.

Two sets of 60 randomly picked Ph-BTBT-C10 dimers were extracted from MD snapshots obtained at $293 \mathrm{~K}$ and $490 \mathrm{~K}$, respectively, for a total of 120 points.

Orbital couplings computed from BLYP/6-31G(d) data were compared with those obtained from much more expensive B3LYP/6-311G(d,p) results.

A scaling factor of $a=1.245$ was obtained as the slope of the fitting line reported in red, in the form $f(x)=a x$. 


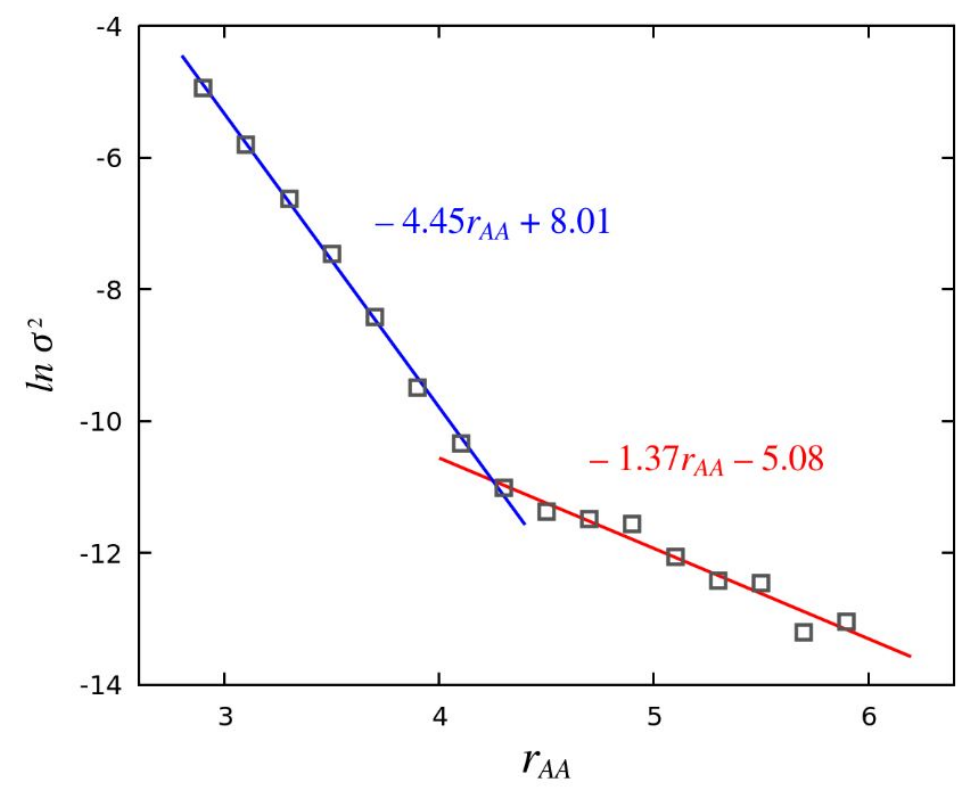

Figure S6. Piecewise exponential fit of $\sigma^{2}\left(r_{A A}\right)\left(\sigma\right.$ in $\mathrm{eV}, r_{A A}$ in $\left.\AA\right)$.

$\sigma^{2}\left(r_{A A}\right)$ is defined as the standard deviation of the subset of orbital couplings associated to a site-to-site spatial displacement $r_{A A}$ binned in $0.2 \AA$ intervals.

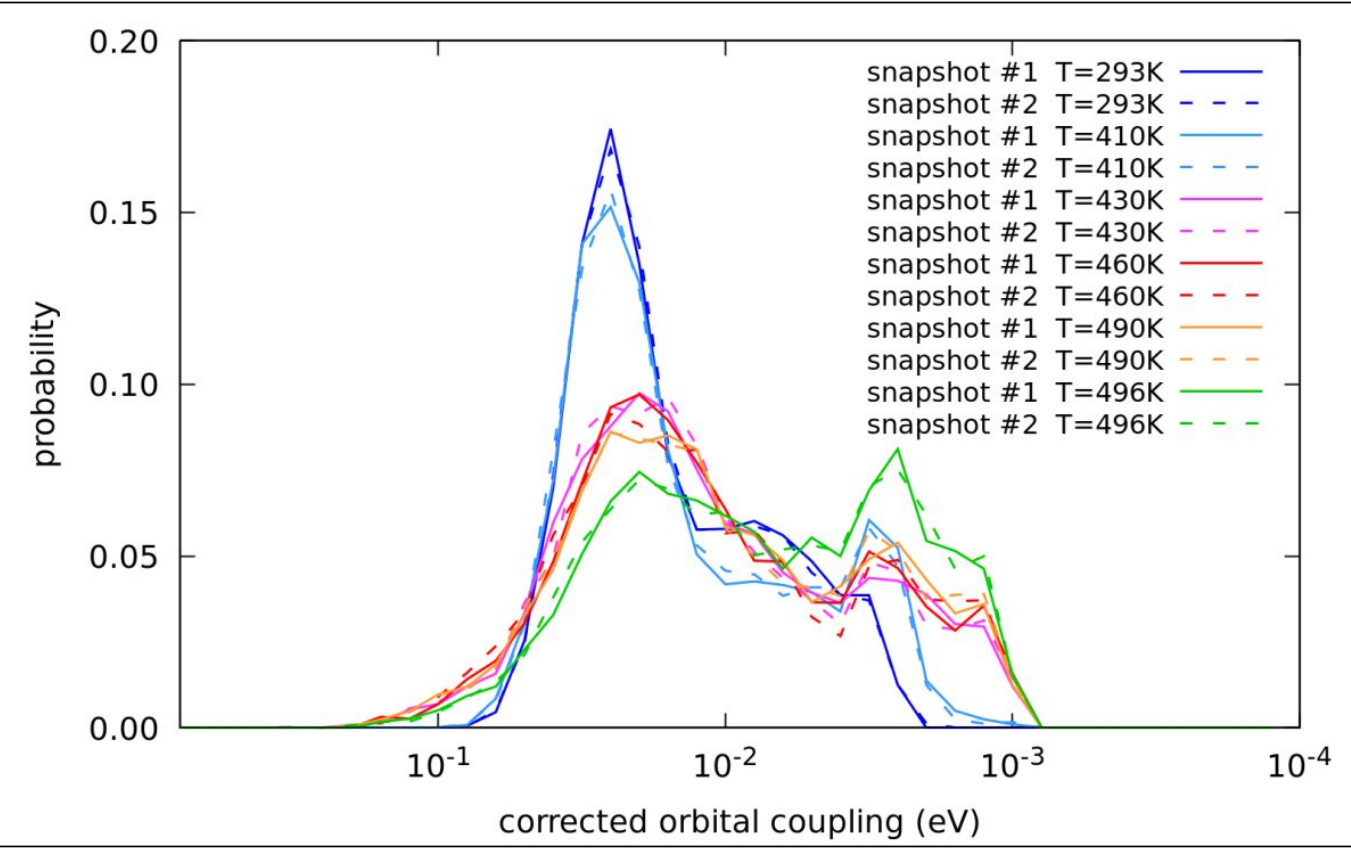

Figure S7. Corrected orbital coupling probability distributions for each molecular structure at each thermodynamic state. 

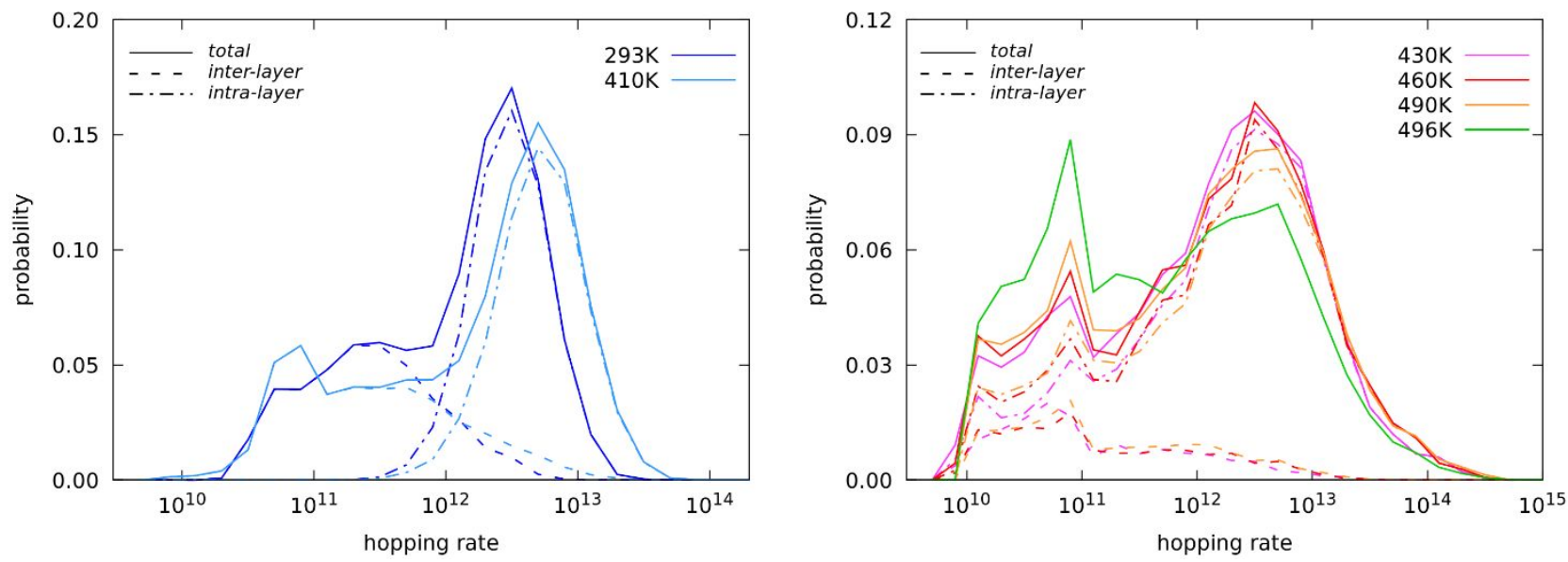

Figure S8. Total, inter-layer, and intra-layer hopping rate probability distributions $\left(\Delta \varepsilon_{\mathrm{pq}}=0\right)$ for crystal (left) and smectic (right) phases. The total distribution for the isotropic phase is also reported (right).

Table S2. Site energy standard deviations (in $\mathrm{meV}$ ).

\begin{tabular}{lcc}
\hline $\mathbf{T}^{a}$ & $\boldsymbol{\sigma}_{\varepsilon, \text { DFT }}^{b}$ & $\boldsymbol{\sigma}_{\varepsilon, \text { Elec }}^{c}$ \\
\hline $\mathbf{2 9 3}$ & 65.3 & 10 \\
$\mathbf{4 1 0}$ & 76.2 & 10 \\
$\mathbf{4 3 0}$ & 77.6 & 110 \\
$\mathbf{4 6 0}$ & 83.2 & 80 \\
$\mathbf{4 9 0}$ & 87.1 & 100 \\
$\mathbf{4 9 6}$ & 86.3 & 100 \\
\hline
\end{tabular}

${ }^{a}$ Temperature $(\mathrm{K})$ of the corresponding thermodynamic state.

${ }^{b}$ Obtained from the distributions of HOMO energies of isolated molecules at BLYP/6-31G(d) level.

${ }^{c}$ Electrostatic disorder, see J. Phys. Chem. Lett. 2015, 6, 3657, or J. Phys. Condens. Matter 2016, 28, 433002. 
Table S3. Hole Diffusivity $\left(10^{-2} \mathrm{~cm}^{2} \mathrm{~s}^{-1}\right)$ as computed from the charge carriers mean square displacement $\left(\Delta \varepsilon_{\mathrm{pq}}=0\right)$ projected along each orientation (identified by angle $\theta$ ) on each $i j$ plane $(x y, x z, y z)$ for each thermodynamic state (identified by the corresponding temperature).

\begin{tabular}{l|c|c|cc|cc|cc|ccc}
\hline \multirow{2}{*}{$\boldsymbol{\theta}^{a}$} & $\mathbf{2 9 3}$ & $\mathbf{4 1 0}$ & \multicolumn{2}{|c|}{$\mathbf{4 3 0}$} & \multicolumn{2}{|c|}{$\mathbf{4 6 0}$} & \multicolumn{2}{|c|}{$\mathbf{4 9 0}$} & \multicolumn{3}{|c}{$\mathbf{4 9 6}$} \\
& $\boldsymbol{x y}$ & $\boldsymbol{x y}$ & $\boldsymbol{x} \boldsymbol{z}$ & $\boldsymbol{x} \boldsymbol{y}$ & $\boldsymbol{x} \boldsymbol{z}$ & $\boldsymbol{x y}$ & $\boldsymbol{x} \boldsymbol{z}$ & $\boldsymbol{x y}$ & $\boldsymbol{x} \boldsymbol{y}$ & $\boldsymbol{x} \boldsymbol{z}$ & $\boldsymbol{y} \boldsymbol{z}$ \\
\hline $\mathbf{0}$ & 1.91 & 3.44 & 1.50 & 1.50 & 1.62 & 1.62 & 1.52 & 1.52 & 1.27 & 1.27 & 1.22 \\
$\mathbf{1 0}$ & 1.89 & 3.41 & 1.49 & 1.49 & 1.59 & 1.62 & 1.49 & 1.52 & 1.28 & 1.26 & 1.21 \\
$\mathbf{2 0}$ & 1.81 & 3.28 & 1.45 & 1.48 & 1.53 & 1.62 & 1.44 & 1.53 & 1.29 & 1.26 & 1.20 \\
$\mathbf{3 0}$ & 1.70 & 3.08 & 1.39 & 1.47 & 1.44 & 1.61 & 1.38 & 1.53 & 1.29 & 1.25 & 1.20 \\
$\mathbf{4 0}$ & 1.56 & 2.82 & 1.31 & 1.46 & 1.33 & 1.60 & 1.30 & 1.53 & 1.28 & 1.24 & 1.20 \\
$\mathbf{5 0}$ & 1.42 & 2.55 & 1.22 & 1.45 & 1.22 & 1.59 & 1.22 & 1.53 & 1.27 & 1.24 & 1.21 \\
$\mathbf{6 0}$ & 1.28 & 2.29 & 1.14 & 1.45 & 1.12 & 1.59 & 1.15 & 1.52 & 1.26 & 1.24 & 1.22 \\
$\mathbf{7 0}$ & 1.17 & 2.08 & 1.07 & 1.45 & 1.04 & 1.58 & 1.09 & 1.52 & 1.25 & 1.25 & 1.23 \\
$\mathbf{8 0}$ & 1.10 & 1.93 & 1.02 & 1.46 & 0.99 & 1.58 & 1.06 & 1.51 & 1.23 & 1.25 & 1.25 \\
$\mathbf{9 0}$ & 1.08 & 1.87 & 1.01 & 1.47 & 0.97 & 1.57 & 1.06 & 1.50 & 1.22 & 1.26 & 1.26 \\
$\mathbf{1 0 0}$ & 1.11 & 1.91 & 1.02 & 1.48 & 1.00 & 1.57 & 1.08 & 1.50 & 1.21 & 1.27 & 1.27 \\
$\mathbf{1 1 0}$ & 1.18 & 2.04 & 1.06 & 1.50 & 1.06 & 1.58 & 1.13 & 1.50 & 1.20 & 1.28 & 1.28 \\
$\mathbf{1 2 0}$ & 1.29 & 2.24 & 1.13 & 1.51 & 1.15 & 1.58 & 1.20 & 1.49 & 1.20 & 1.29 & 1.28 \\
$\mathbf{1 3 0}$ & 1.43 & 2.49 & 1.21 & 1.52 & 1.26 & 1.59 & 1.28 & 1.49 & 1.21 & 1.29 & 1.28 \\
$\mathbf{1 4 0}$ & 1.58 & 2.76 & 1.29 & 1.52 & 1.37 & 1.60 & 1.36 & 1.50 & 1.22 & 1.29 & 1.27 \\
$\mathbf{1 5 0}$ & 1.71 & 3.02 & 1.37 & 1.53 & 1.48 & 1.61 & 1.43 & 1.50 & 1.23 & 1.29 & 1.26 \\
$\mathbf{1 6 0}$ & 1.82 & 3.24 & 1.44 & 1.52 & 1.56 & 1.61 & 1.48 & 1.50 & 1.24 & 1.29 & 1.25 \\
$\mathbf{1 7 0}$ & 1.89 & 3.39 & 1.49 & 1.51 & 1.61 & 1.62 & 1.51 & 1.51 & 1.26 & 1.28 & 1.23 \\
\hline
\end{tabular}

${ }^{a}$ Reported in degrees.

${ }^{b}$ Temperature $(\mathrm{K})$ of the corresponding thermodynamic state. 
Table S4. Standard deviation of hole Diffusivity as computed from the charge carriers mean square displacement $\left(\Delta \varepsilon_{\mathrm{pq}}=0\right)$ projected along each orientation (identified by angle $\theta$ ) on each $i j$ plane $(x y, x z, y z)$ for each thermodynamic state (identified by the corresponding temperature). Values are expressed as a percentage of the corresponding average Diffusivity in the same $\theta, i j, \mathrm{~T}$ conditions. Plan-wise (in red, superscript $P$ ) and $\theta$-wise (in blue, superscript $\theta$ ) average and maximum values are reported.

\begin{tabular}{|c|c|c|c|c|c|c|c|c|c|c|c|c|c|}
\hline \multirow{2}{*}{$\boldsymbol{\theta}^{a}$} & \multirow{2}{*}{$\begin{array}{r}293^{b} \\
x y \\
\end{array}$} & \multirow{2}{*}{$\begin{array}{c}410 \\
x y\end{array}$} & \multicolumn{2}{|c|}{430} & \multicolumn{2}{|c|}{460} & \multicolumn{2}{|c|}{490} & \multicolumn{3}{|c|}{496} & \multirow{2}{*}{${ }^{\theta}$ avg } & \multirow{2}{*}{${ }^{\theta} \max$} \\
\hline & & & $x z$ & $x y$ & $x z$ & $x y$ & $x z$ & $x y$ & $x y$ & $x z$ & $y z$ & & \\
\hline $\mathbf{0}$ & 0.21 & 0.47 & 0.56 & 0.56 & 0.56 & 0.56 & 0.28 & 0.28 & 0.18 & 0.18 & 1.25 & 0.46 & 1.25 \\
\hline 10 & 0.34 & 0.50 & 0.60 & 0.50 & 0.58 & 0.44 & 0.46 & 0.29 & 0.19 & 0.29 & 0.99 & 0.47 & 0.99 \\
\hline 20 & 0.55 & 0.50 & 0.71 & 0.47 & 0.60 & 0.29 & 0.65 & 0.32 & 0.18 & 0.40 & 0.68 & 0.49 & 0.71 \\
\hline 30 & 0.81 & 0.47 & 0.88 & 0.54 & 0.59 & 0.14 & 0.81 & 0.37 & 0.24 & 0.48 & 0.39 & 0.52 & 0.88 \\
\hline 40 & 1.09 & 0.41 & 1.10 & 0.72 & 0.57 & 0.19 & 0.93 & 0.45 & 0.39 & 0.51 & 0.19 & 0.60 & 1.10 \\
\hline 50 & 1.37 & 0.34 & 1.33 & 0.94 & 0.54 & 0.34 & 0.99 & 0.56 & 0.60 & 0.49 & 0.23 & 0.70 & 1.37 \\
\hline 60 & 1.61 & 0.29 & 1.54 & 1.14 & 0.51 & 0.47 & 0.97 & 0.68 & 0.81 & 0.42 & 0.30 & 0.80 & 1.61 \\
\hline 70 & 1.75 & 0.36 & 1.67 & 1.27 & 0.53 & 0.55 & 0.86 & 0.80 & 1.01 & 0.31 & 0.30 & 0.86 & 1.75 \\
\hline 80 & 1.73 & 0.51 & 1.69 & 1.31 & 0.61 & 0.56 & 0.66 & 0.91 & 1.16 & 0.20 & 0.21 & 0.87 & 1.73 \\
\hline 90 & 1.53 & 0.63 & 1.58 & 1.26 & 0.69 & 0.51 & 0.41 & 0.98 & 1.25 & 0.16 & 0.16 & 0.83 & 1.58 \\
\hline 100 & 1.18 & 0.67 & 1.35 & 1.11 & 0.75 & 0.40 & 0.18 & 1.01 & 1.25 & 0.23 & 0.35 & 0.77 & 1.35 \\
\hline 110 & 0.79 & 0.62 & 1.06 & 0.90 & 0.75 & 0.26 & 0.17 & 0.99 & 1.18 & 0.32 & 0.63 & 0.70 & 1.18 \\
\hline 120 & 0.46 & 0.50 & 0.79 & 0.66 & 0.70 & 0.19 & 0.28 & 0.92 & 1.03 & 0.39 & 0.93 & 0.62 & 1.03 \\
\hline 130 & 0.27 & 0.38 & 0.61 & 0.44 & 0.63 & 0.29 & 0.33 & 0.81 & 0.82 & 0.42 & 1.19 & 0.56 & 1.19 \\
\hline 140 & 0.24 & 0.30 & 0.54 & 0.33 & 0.57 & 0.44 & 0.31 & 0.67 & 0.60 & 0.40 & 1.39 & 0.53 & 1.39 \\
\hline 150 & 0.25 & 0.31 & 0.53 & 0.40 & 0.54 & 0.56 & 0.23 & 0.53 & 0.38 & 0.33 & 1.50 & 0.51 & 1.50 \\
\hline 160 & 0.23 & 0.36 & 0.54 & 0.50 & 0.53 & 0.62 & 0.13 & 0.40 & 0.22 & 0.24 & 1.52 & 0.48 & 1.52 \\
\hline 170 & 0.19 & 0.42 & 0.55 & 0.56 & 0.54 & 0.63 & 0.13 & 0.31 & 0.17 & 0.16 & 1.43 & 0.46 & 1.43 \\
\hline${ }^{P}$ avg & 0.81 & 0.45 & 0.98 & 0.76 & 0.60 & 0.41 & 0.49 & 0.63 & 0.65 & 0.33 & 0.76 & $0.62^{c}$ & \\
\hline${ }^{P_{\max }}$ & 1.75 & 0.67 & 1.69 & 1.31 & 0.75 & 0.63 & 0.99 & 1.01 & 1.25 & 0.51 & 1.52 & & $1.75^{d}$ \\
\hline
\end{tabular}

${ }^{a}$ Reported in degrees.

${ }^{b}$ Temperature $(\mathrm{K})$ of the corresponding thermodynamic state.

${ }^{c}$ Average relative standard deviation extended to all orientations in all planes at each thermodynamic state.

${ }^{d}$ Maximum relative standard deviation extended to all orientations in all planes at each thermodynamic state. 
Table S5. Hole Diffusivity $\left(10^{-3} \mathrm{~cm}^{2} \mathrm{~s}^{-1}\right)$ as computed from the charge carriers mean square displacement $\left(\Delta \varepsilon_{\mathrm{pq}} \neq 0\right)$ projected along each orientation (identified by angle $\left.\theta\right)$ on each $i j$ plane $(x y, x z, y z)$ for each thermodynamic state (identified by the corresponding temperature).

\begin{tabular}{l|c|c|cc|cc|cc|ccc}
\hline \multirow{2}{*}{$\boldsymbol{\theta}^{a}$} & $\mathbf{2 9 3}$ & $\mathbf{4 1 0}$ & \multicolumn{2}{|c|}{$\mathbf{4 3 0}$} & \multicolumn{2}{|c|}{$\mathbf{4 6 0}$} & \multicolumn{2}{|c|}{$\mathbf{4 9 0}$} & \multicolumn{3}{|c}{$\mathbf{4 9 6}$} \\
& $\boldsymbol{x y}$ & $\boldsymbol{x y}$ & $\boldsymbol{x} \boldsymbol{z}$ & $\boldsymbol{x} \boldsymbol{y}$ & $\boldsymbol{x} \boldsymbol{z}$ & $\boldsymbol{x y}$ & $\boldsymbol{x} \boldsymbol{z}$ & $\boldsymbol{x y}$ & $\boldsymbol{x} \boldsymbol{y}$ & $\boldsymbol{x} \boldsymbol{z}$ & $\boldsymbol{y} \boldsymbol{z}$ \\
\hline $\mathbf{0}$ & 1.30 & 4.61 & 1.83 & 1.83 & 1.86 & 1.86 & 1.79 & 1.79 & 1.74 & 1.74 & 1.72 \\
$\mathbf{1 0}$ & 1.28 & 4.51 & 1.82 & 1.82 & 1.84 & 1.87 & 1.77 & 1.80 & 1.77 & 1.73 & 1.72 \\
$\mathbf{2 0}$ & 1.23 & 4.29 & 1.78 & 1.80 & 1.80 & 1.87 & 1.73 & 1.81 & 1.79 & 1.72 & 1.72 \\
$\mathbf{3 0}$ & 1.16 & 3.99 & 1.72 & 1.79 & 1.73 & 1.86 & 1.67 & 1.82 & 1.81 & 1.72 & 1.72 \\
$\mathbf{4 0}$ & 1.07 & 3.62 & 1.65 & 1.78 & 1.64 & 1.84 & 1.61 & 1.83 & 1.81 & 1.72 & 1.73 \\
$\mathbf{5 0}$ & 0.98 & 3.24 & 1.58 & 1.77 & 1.55 & 1.82 & 1.55 & 1.84 & 1.81 & 1.74 & 1.75 \\
$\mathbf{6 0}$ & 0.89 & 2.90 & 1.51 & 1.77 & 1.47 & 1.80 & 1.50 & 1.85 & 1.80 & 1.75 & 1.76 \\
$\mathbf{7 0}$ & 0.82 & 2.63 & 1.45 & 1.77 & 1.40 & 1.78 & 1.47 & 1.85 & 1.78 & 1.77 & 1.78 \\
$\mathbf{8 0}$ & 0.77 & 2.46 & 1.42 & 1.78 & 1.35 & 1.76 & 1.45 & 1.85 & 1.75 & 1.79 & 1.80 \\
$\mathbf{9 0}$ & 0.76 & 2.43 & 1.41 & 1.80 & 1.34 & 1.74 & 1.45 & 1.85 & 1.72 & 1.81 & 1.81 \\
$\mathbf{1 0 0}$ & 0.77 & 2.52 & 1.42 & 1.81 & 1.36 & 1.73 & 1.48 & 1.84 & 1.70 & 1.83 & 1.82 \\
$\mathbf{1 1 0}$ & 0.82 & 2.74 & 1.46 & 1.82 & 1.41 & 1.74 & 1.52 & 1.83 & 1.68 & 1.84 & 1.82 \\
$\mathbf{1 2 0}$ & 0.89 & 3.05 & 1.52 & 1.84 & 1.48 & 1.74 & 1.57 & 1.81 & 1.66 & 1.84 & 1.82 \\
$\mathbf{1 3 0}$ & 0.98 & 3.42 & 1.59 & 1.85 & 1.56 & 1.76 & 1.63 & 1.80 & 1.66 & 1.83 & 1.81 \\
$\mathbf{1 4 0}$ & 1.07 & 3.79 & 1.66 & 1.85 & 1.65 & 1.78 & 1.69 & 1.79 & 1.66 & 1.82 & 1.79 \\
$\mathbf{1 5 0}$ & 1.16 & 4.14 & 1.73 & 1.85 & 1.74 & 1.80 & 1.74 & 1.79 & 1.67 & 1.80 & 1.77 \\
$\mathbf{1 6 0}$ & 1.23 & 4.41 & 1.78 & 1.85 & 1.80 & 1.83 & 1.78 & 1.78 & 1.69 & 1.78 & 1.75 \\
$\mathbf{1 7 0}$ & 1.28 & 4.57 & 1.82 & 1.84 & 1.85 & 1.85 & 1.79 & 1.79 & 1.72 & 1.76 & 1.74 \\
\hline
\end{tabular}

${ }^{a}$ Reported in degrees.

${ }^{b}$ Temperature $(\mathrm{K})$ of the corresponding thermodynamic state. 
Table S6. Standard deviation of hole Diffusivity as computed from the charge carriers mean square displacement $\left(\Delta \varepsilon_{\mathrm{pq}} \neq 0\right)$ projected along each orientation (identified by angle $\theta$ ) on each $i j$ plane $(x y, x z, y z)$ for each thermodynamic state (identified by the corresponding temperature). Values are expressed as a percentage of the corresponding average Diffusivity in the same $\theta, i j, \mathrm{~T}$ conditions. Plan-wise (in red, superscript $P$ ) and $\theta$-wise (in blue, superscript $\theta$ ) average and maximum values are reported.

\begin{tabular}{|c|c|c|c|c|c|c|c|c|c|c|c|c|c|}
\hline \multirow{2}{*}{$\boldsymbol{\theta}^{a}$} & \multirow{2}{*}{$\begin{array}{c}2^{293} \\
x y \\
\end{array}$} & \multirow{2}{*}{$\begin{array}{c}410 \\
x y \\
\end{array}$} & \multicolumn{2}{|c|}{430} & \multicolumn{2}{|c|}{460} & \multicolumn{2}{|c|}{490} & \multicolumn{3}{|c|}{496} & \multirow{2}{*}{${ }^{\theta}$ avg } & \multirow{2}{*}{${ }^{\theta} \max$} \\
\hline & & & $x z$ & $x y$ & $x z$ & $x y$ & $x z$ & $x y$ & $x y$ & $x z$ & $y z$ & & \\
\hline $\mathbf{0}$ & 1.48 & 0.29 & 0.33 & 0.33 & 0.20 & 0.20 & 1.25 & 1.25 & 0.33 & 0.33 & 0.29 & 0.57 & 1.48 \\
\hline 10 & 1.48 & 0.44 & 0.25 & 0.48 & 0.13 & 0.29 & 1.39 & 1.07 & 0.46 & 0.34 & 0.28 & 0.60 & 1.48 \\
\hline 20 & 1.40 & 0.66 & 0.21 & 0.64 & 0.15 & 0.37 & 1.51 & 0.85 & 0.65 & 0.37 & 0.28 & 0.64 & 1.51 \\
\hline 30 & 1.23 & 0.96 & 0.19 & 0.76 & 0.30 & 0.40 & 1.62 & 0.63 & 0.82 & 0.42 & 0.31 & 0.69 & 1.62 \\
\hline 40 & 0.98 & 1.32 & 0.18 & 0.84 & 0.48 & 0.38 & 1.68 & 0.43 & 0.92 & 0.49 & 0.40 & 0.74 & 1.68 \\
\hline 50 & 0.64 & 1.72 & 0.23 & 0.86 & 0.67 & 0.32 & 1.69 & 0.31 & 0.93 & 0.57 & 0.52 & 0.77 & 1.72 \\
\hline 60 & 0.26 & 2.09 & 0.40 & 0.83 & 0.85 & 0.26 & 1.63 & 0.30 & 0.86 & 0.67 & 0.65 & 0.80 & 2.09 \\
\hline 70 & 0.23 & 2.37 & 0.62 & 0.74 & 0.98 & 0.28 & 1.52 & 0.34 & 0.71 & 0.77 & 0.77 & 0.85 & 2.37 \\
\hline 80 & 0.55 & 2.43 & 0.87 & 0.64 & 1.05 & 0.41 & 1.35 & 0.40 & 0.50 & 0.85 & 0.85 & 0.90 & 2.43 \\
\hline 90 & 0.73 & 2.23 & 1.10 & 0.54 & 1.04 & 0.57 & 1.17 & 0.47 & 0.29 & 0.90 & 0.90 & 0.90 & 2.23 \\
\hline 100 & 0.69 & 1.80 & 1.26 & 0.47 & 0.96 & 0.70 & 1.00 & 0.58 & 0.27 & 0.91 & 0.90 & 0.87 & 1.80 \\
\hline 110 & 0.47 & 1.27 & 1.32 & 0.45 & 0.82 & 0.79 & 0.89 & 0.74 & 0.47 & 0.89 & 0.85 & 0.82 & 1.32 \\
\hline 120 & 0.23 & 0.77 & 1.30 & 0.46 & 0.68 & 0.81 & 0.83 & 0.93 & 0.67 & 0.83 & 0.77 & 0.75 & 1.30 \\
\hline 130 & 0.40 & 0.38 & 1.19 & 0.46 & 0.55 & 0.77 & 0.83 & 1.11 & 0.79 & 0.74 & 0.66 & 0.72 & 1.19 \\
\hline 140 & 0.73 & 0.13 & 1.03 & 0.42 & 0.46 & 0.66 & 0.86 & 1.27 & 0.82 & 0.63 & 0.54 & 0.69 & 1.27 \\
\hline 150 & 1.02 & 0.12 & 0.84 & 0.35 & 0.40 & 0.50 & 0.93 & 1.38 & 0.75 & 0.52 & 0.43 & 0.66 & 1.38 \\
\hline 160 & 1.25 & 0.19 & 0.65 & 0.27 & 0.34 & 0.34 & 1.02 & 1.42 & 0.59 & 0.43 & 0.35 & 0.62 & 1.42 \\
\hline 170 & 1.40 & 0.23 & 0.47 & 0.24 & 0.28 & 0.21 & 1.13 & 1.37 & 0.41 & 0.36 & 0.31 & 0.58 & 1.40 \\
\hline${ }^{P}$ avg & 0.84 & 1.08 & 0.69 & 0.54 & 0.57 & 0.46 & 1.24 & 0.83 & 0.63 & 0.61 & 0.56 & $\mathbf{0 . 7 3}^{c}$ & \\
\hline${ }^{P_{\max }}$ & 1.48 & 2.43 & 1.32 & 0.86 & 1.05 & 0.81 & 1.69 & 1.42 & 0.93 & 0.91 & 0.90 & & $2.43^{d}$ \\
\hline
\end{tabular}

${ }^{a}$ Reported in degrees.

${ }^{b}$ Temperature $(\mathrm{K})$ of the corresponding thermodynamic state.

${ }^{c}$ Average relative standard deviation extended to all orientations in all planes at each thermodynamic state.

${ }^{d}$ Maximum relative standard deviation extended to all orientations in all planes at each thermodynamic state. 

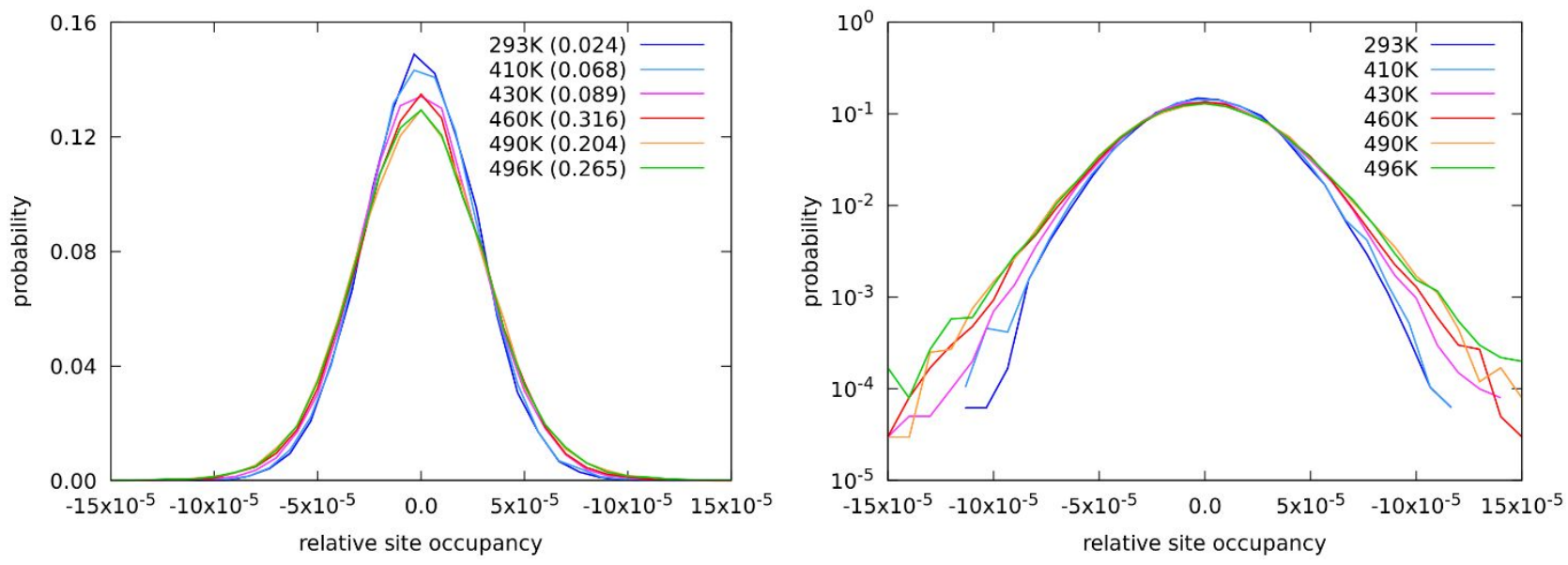

Figure S9. Relative site occupancy probability distributions $\left(\Delta \varepsilon_{\mathrm{pq}}=0\right)$ for each thermodynamic state, on a linear (left) and logarithmic (right) axis. Brackets in the left-hand panel report non-Gaussianity parameters for each distribution.

In the absence of significant geometrical bias related to the starting structures, the fraction of total simulation time spent by the charge on any given site would give a Gaussian distribution centered at $\mathrm{N}_{S}^{-1}$, with $\mathrm{N}_{S}$ the number of unique sites in the simulation. Fig. S9 shows site occupancy probability distributions for each thermodynamic state considered. Since fractional site occupancy is an extensive property of the system (related to $\mathrm{N}_{S}$ ), these distributions are translated by $\mathrm{N}_{S}^{-1}$ (hence, the "relative" site occupancy label). Relative distributions indeed peak at zero, confirming the reliability of the starting structures. However, although unimodal, these distributions lose Gaussian character as the corresponding simulation temperature increases. This can be seen on the logscale plot, as tails divert from the expected parabolic behavior of an ideal Gaussian curve, extending instead seemingly-linearly at higher temperatures. The divergence from a Gaussian distribution was quantified via the non-Gaussianity parameter, defined as:

$$
\alpha=\frac{\left\langle\Delta r^{4}\right\rangle}{3\left\langle\Delta r^{2}\right\rangle^{2}}-1
$$

For a truly Gaussian distribution in $\Delta r, \alpha$ should equal zero. The positive values of $\alpha$ obtained indicate indeed a slightly wider-than-Gaussian distribution. This entails the presence of anomalously high probabilities far away from the peak on either side. However, these could either correspond to seldom-visited sites featuring rather low average transfer rates, or to extremely active sites characterized by high average transfer rates. This is most relevant within distribution tails, but is nonetheless true for any point of the probability distributions reported. 


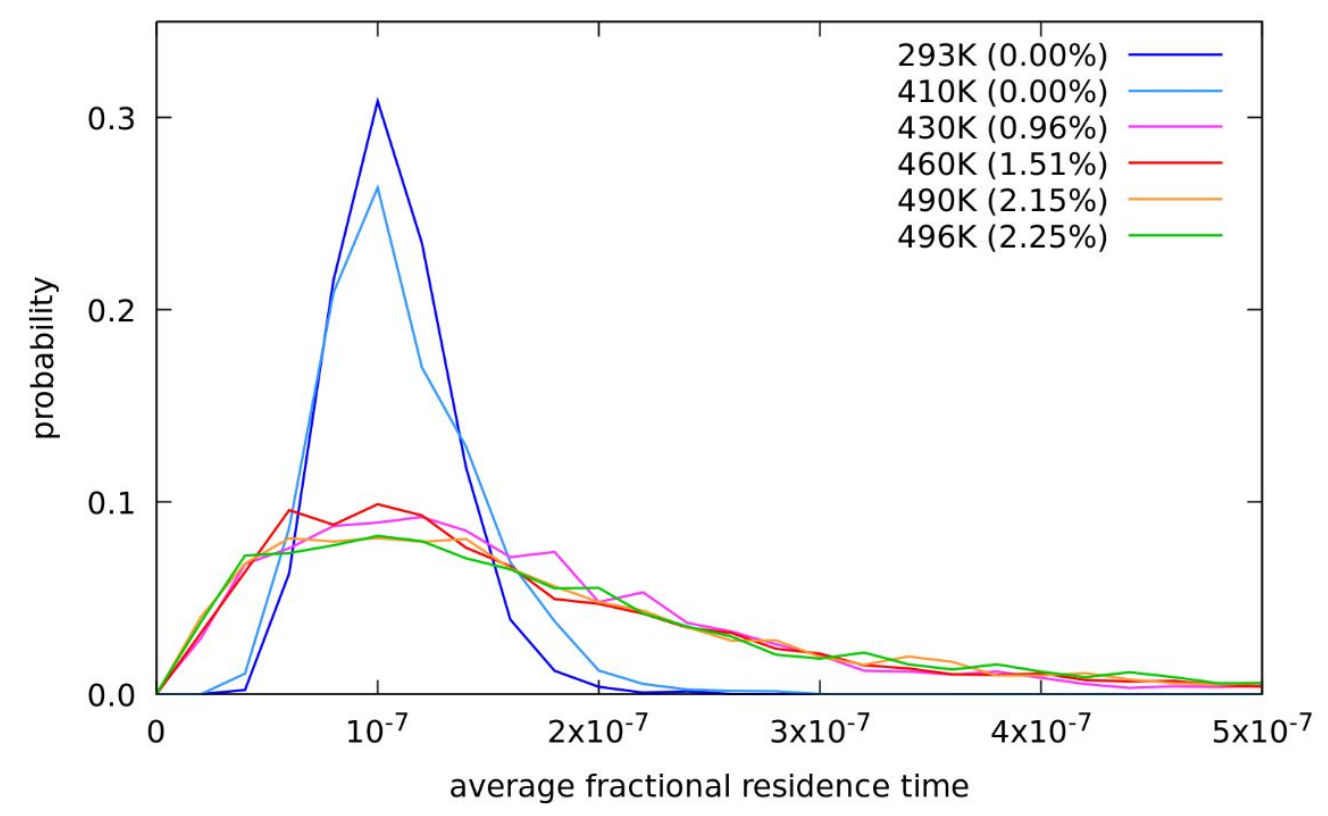

Figure S10. Average residence time probability distributions $\left(\Delta \varepsilon_{\mathrm{pq}}=0\right)$. In brackets, the percentage of sites with an average residence time longer than $5 \times 10^{-7}$

An average residence time for each site was obtained as the total residence time of a charge on that site, normalized over the total simulation time, and divided by the number of times that site was "visited" during the simulation. The corresponding probability distributions are reported in Fig. S10. These peak at $n_{\text {steps }}^{-1}$ for both crystalline and smectic states, with $n_{\text {steps }}$ the total number of KMC steps (in the neighborhood of $10^{7}$ ). This is the expected value for an homogeneous percolation network in which the time lapse intervening between two consecutive hops is independent of the sites involved. However, while the crystalline phase affords sharp probability distributions, slightly tailed towards higher residence times, smectic states present broader distributions with extended tails, entailing the presence of "trap" sites experiencing average residence times much longer than average. 

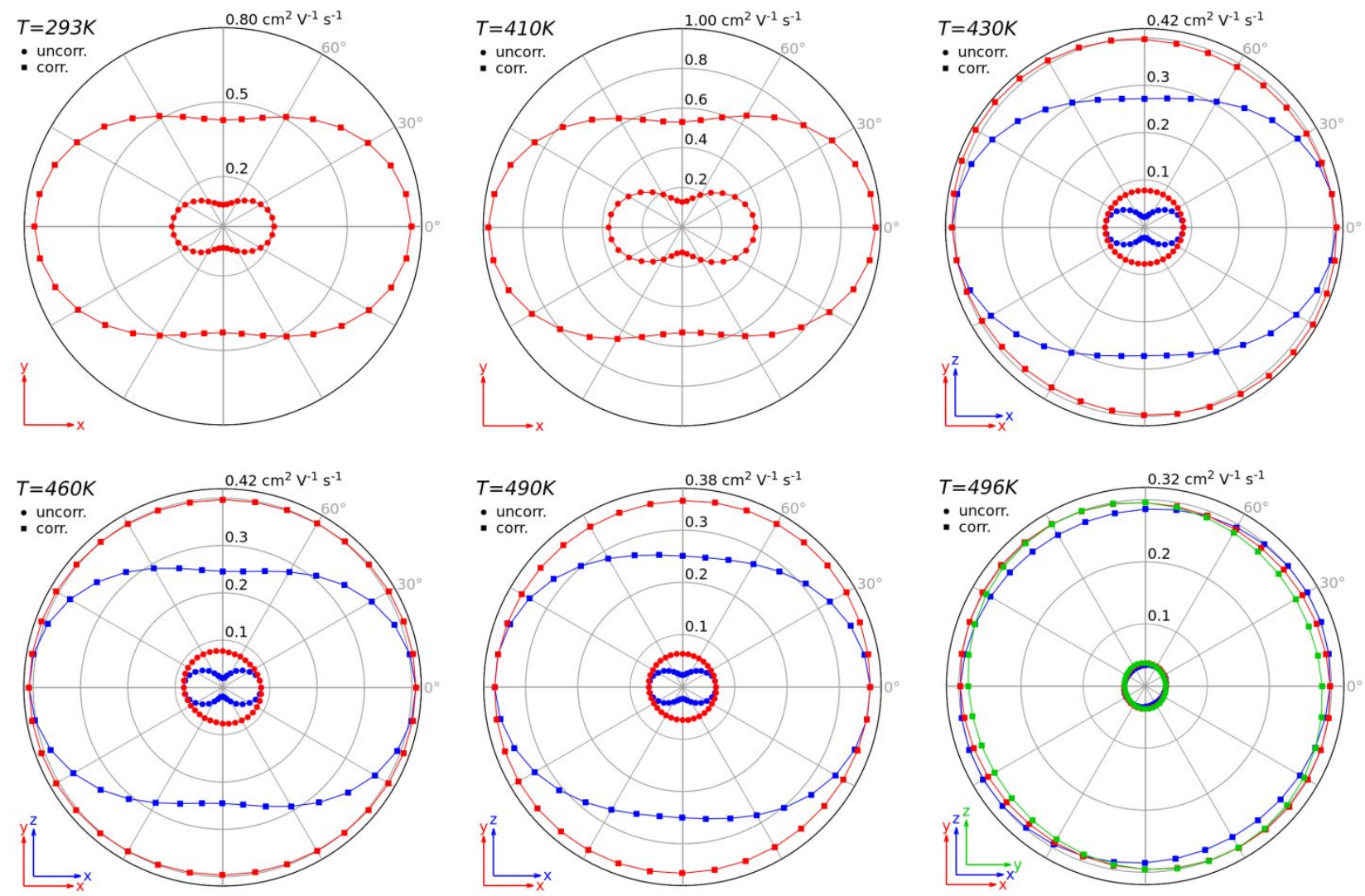

Figure S11. Hole mobilities projected onto relevant planes for the six thermodynamic states, in the absence of energetic disorder $\left(\Delta \varepsilon_{\mathrm{pq}}=0\right)$. Squares (corr. data series) and circles (uncorr. data series) represent mobility estimates obtained from QM-based orbital couplings, scaled to B3LYP/6-311G(d,p) reference by a factor of 1.245 (see Figure S5 for details), with and without correction for dynamic disorder (see Figure S6).

The introduction of dynamic disorder evidently reduces anisotropy across the board. This effect is only slightly affected by simulation temperature. Comparison of the two sets of data, corrected and uncorrected, however, shows the effects of the piecewise correction on the different morphologies. The hole mobility of room temperature crystal morphologies $(293 \mathrm{~K})$ increases 4 -fold upon correction. A similar increase, by a factor of 4-4.5, is obtained at low temperature smectic states $(430 \mathrm{~K}$ and $460 \mathrm{~K})$. As temperature increases to the smectic-isotropic transition, the effect of the correction is further enhanced, with increases of a factor of 5 at $490 \mathrm{~K}$ (prior to the transition) and of a factor of 10 at $496 \mathrm{~K}$ (onset of the isotropic phase).

Anomalous, in this context, is the behavior of hole mobility estimates in the high temperature region of the crystal state $(410 \mathrm{~K})$, which yield an increase of only 2.5 times upon introduction of dynamic off-diagonal disorder. This result can however be explained by the large values obtained for orbital couplings at such temperature prior to any correction due to local excess thermal energy in a well-order herringbone-like crystal morphology, allowing for large displacement around equilibrium positions and thus close contacts between neighboring molecules. Due to the additive nature of the correction used, its effect is bound to be diminished the larger the quantity to be corrected grows. 